J. Clin. Chem. Clin. Biochem.

Vol. 16, 1978, pp. 587-595

\title{
Untersuchungen zur Vergleichbarkeit der Ergebnisse unterschiedlicher Methoden zur radioimmunologischen Digoxin-Bestimmung Ein Beitrag zur Qualitätskontrolle
}

\author{
Von A. Dwenger und I. Trautschold \\ Institut für Klinische Biochemie der Medizinischen Hochschule Hannover
}

(Eingegangen am 17. April/6. Juli 1978)

Zusammenfassung: Unter Verwendung von drei Testkombinationen zur radioimmunòlogischen Digoxin-Bestimmung (A Amersham Buchler; B Boehringer Mannheim; C Schwarz Mann/Becton Dickinson) werden Ergebnisse zur Qualität und Vergleichbarkeit der Methoden beschrieben. Durch Ermittlung der Antigen-Konzentrationen in Proben unterschiedlicher Medien (a Poolserum; b artefizielles Humanserum; c Albumin- und Globulinhaltige Pufferlösung), in Seren aus einem pharmakokinetischen Versuch mit Digoxin sowie in Seren mit unterschiedlichen Protein-Konzentrationen, mit Hämolyse, Digitoxin und Spironolacton-Metaboliten werden intra- und inter-assay Präzisionen berechnet.

Die intra-assay Präzision wird vom Medium der Proben beeinflußt. Sie ist für Proben, bei denen eine identische Digoxin-Konzentration im gleichen Medium vorliegt, besser (VK für $2 \mu \mathrm{g} / \mathrm{l}$ in z. B. Medium a für Testkombination A: 4,3\%; für B: 7,0\%; für C: $2,2 \%$ ) als für Proben, bei denen das Antigen in unterschiedlichen Medien vorliegt (VK für $2 \mu \mathrm{g} / 1$ in den Medien a, b und c für Testkombination A: 6,4\%; für B: 9,1\%; für C: 4,3\%).

Die mittleren Wiederfinderaten über den Konzentrations-Bereich 0,5-4 $\mu \mathrm{g} / 1$ variieren in Abhängigkeit von den Medien a, b und c für die Testkombination A von $84,4 \%$ bis $100,8 \%$, für B von $112,0 \%$ bis $119,6 \%$, für C von $98,0 \%$ bis $104,5 \%$.

Eine Abnahme der Gesamt-Serumprotein-Konzentration auf weniger als die Hälfte führt bei der Testkombination A zu falsch negativen, bei $\mathrm{C}$ zu falsch positiven Ergebnissen; bei B wird eine derartige Abhängigkeit nicht beobachtet, dagegen eine Zunahme der Streuungen.

Hämolyse (Hämoglobin zugesetzt: 1,2 g/1) bewirkt bei Testkombination B eine geringfügige Erhöhung der ermittelten Konzentration.

Digitoxin und Spironolacton-Metabolite täuschen in Abhängigkeit der individuellen Antikörper-Spezifitäten der Testkombinationen Digoxin-Konzentrationen in unterschiedlichem Ausmaße vor, wobei der Antikörper der Testkombination B nicht mit Spironolacton-Metaboliten kreuzreagiert.

Die Berücksichtigung der individuellen unspezifischen Bindungen beeinflußt die Ergebnisse nicht, da die unspezifischen Bindungen für Proben und Standards identisch sind.

Durch Verwendung der Wiederfindekurven (Digoxin in den Medien a, b oder c) als Bezugs-Standardkurven kann jeweils eine Verbesserung der inter-assay Präzision um nahezu das Doppelte demonstriert werden; mögliche Auswirkungen auf die Verbesseerung der Vergleichbarkeit der Ergebnisse aus radioimmunologischen Digoxin-Bestimmungen werden diskutiert.

\section{Studies on the comparability of the results from different methods} for the radioimmunological determination of digoxin: a contribution to quality control

Summary: Three iodine-125-digoxin radioimmunoassay kits (A Amersham Buchler; B Boehringer Mannheim; C Schwarz Mann/Becton Dickinson) were evaluated with respect to assay quality and comparability of the results. Intra- and interassay variances were calculated for the following types of samples: Three media (a pool serum; $b$ artificial human serum; $c$ buffer solution with albumin and globulin) containing pure digoxin, sera from a pharmacokinetic study, sera with different concentrations of proteins, a hemolytic serum and sera with digitoxin and metabolites of spironolactone. 
The intra-assay precision depended on the medium of the sample and was higher for samples with identical digoxin concentrations in an identical medium (e.g. CV for $2 \mu \mathrm{g} / 1$ in medium a for kit A: $4.3 \%$; for kit B: $7.0 \%$; for kit C: $2.2 \%$ ) than for samples with identical antigen concentrations in different media (CV for $2 \mu \mathrm{g} / 1$ in media a, b and $\mathrm{c}$ for kit A: $6.4 \%$; for kit B: $9.1 \%$; for kit C: $4.3 \%$ ).

The mean recovery in the range of $0.5-4 \mu \mathrm{g} / 1$ depended on the kind of medium $(\mathrm{a}, \mathrm{b}$ or $\mathrm{c}$ ) and varied for kit $\mathrm{A}$ from $84.4 \%$ to $100.8 \%$, for kit B from $112.0 \%$ to $119.6 \%$, and for kit C from $98.0 \%$ to $104.5 \%$.

Decreasing serum protein concentrations to less than one half of the physiological concentration gave false negative results for kit A and false positive results for kit C; for kit B this dependency was not be observed, but there was a decrease of reproducibility.

Moderate hemolysis (hemoglobin added: $1.2 \mathrm{~g} / \mathrm{l}$ ) caused a slight increase of values from kit $\mathrm{B}$.

In contrast to the antiserum of kit $B$, which only cross-reacted with digitoxin, the antisera of kits $A$ and $C$ also reacted with metabolites of spironolactone, thereby feigning digoxin concentrations up to $2.8 \mu \mathrm{g} / \mathrm{l}$.

Since the individual unspecific bindings for standards and samples were identical, no improvement of the results was observed after correction of the values for individual unspecific bindings.

By using each of the recovery curves in the media a, b or $\mathrm{c}$ instead of the kit standards, a considerable improvement of the inter-assay precision was demonstrated; possible consequences of the improvement of the comparability of the results from different methods for the digoxin radioimmunoassay are discussed.

\section{Einleitung}

Unmittelbar nach Einfuihrung der radioimmunologischen Digoxin-Bestimmung (1) konnte eine stetige Zunahme der Bestimmungs-Häufigkeit beobachtet werden, der entsprechend der allgemeinen Entwicklung auf radioimmunologischem Gebiet die Einführung und Verwendung neuer konfektionierter Testkombinationen folgte. Auswirkungen auf die Vergleichbarkeit von Ergebnissen blieben hierbei nicht aus, in vergleichenden Untersuchungen einiger der momentan etwa 20 verschiedenen kommerziell vertriebenen Testkits sind Einflüsse unterschiedlicher Art beschrieben worden (2-23). Die Ergebnisse des ersten in der Bundesrepublik Deutschland durchgeführten Digoxin-Ringversuches (24) zeigten, daß Unterschiede zwischen den Methoden bei der radioimmunologischen Digoxin-Bestimmung zwar geringere Auswirkungen auf die Ergebnisse haben als bei HormonBestimmungen (25-33), daß die Präzision zwischen den Methoden aber diejenige anderer klinisch-chemischer oder biochemischer Analysen-Verfahren nicht erreicht (37). Das auch bei dem Digoxin-Ringversuch (24) angewandte, erstmalig von Erhardt et al (27) und Marschner et al (25) beschriebene und inzwischen wiederholt praktizierte Verfahren, in antigenarmen oder -freien Seren angesetzte Standards als uniforme Standardkurve (Wiederfindekurve) zu verwenden, hat wie bei allen in dieser Weise konzipierten Ringversuchen $(25,26,28,29)$ gezeigt, daß sich hiermit eine generelle und teilweise außerordentliche Verbesserung der Präzision zwischen den Laboratorien bzw. zwischen den Methoden erreichen läßt. Als wesentliche Konsequenz für die Qualitätskontrolle wird empfohlen, Standardkurven für radioimmunologische Hormon-Bestimmungen in antigenarmen Seren anzusetzen $(25-29,33-35)$.
Für die radioimmunologische Digoxin-Bestimmung fehlen bisher Hinweise, ob die für die Hormon-Bestimmungen diskutierte Ausschaltung von Tracer-Artefakten durch Verwendung nativen Serums zur Herstellung von Standardlösungen auch vorrangig an der Verbesserung der Präzision beteiligt ist. $\mathrm{Da}$ bei der Digoxin-Bestimmung die Art der Medien, in denen das Standard-Antigen enthalten ist (natives Mischserum, stabilisiertes Humanserum, rekonstituiertes Serum-Lyophilisat, Pufferlösungen, alkoholische Lösungen), in den kommerziell vertriebenen Testkombinationen unterschiedlich isti; kann nicht ausgeschlossen werden, daß die Verbesserung der Präzision hierbei auf den Charakter der Einheitlichkeit der Bezugs-Standardkurve (Wiederfindekurve) zurückzufuihren ist. Dadurch werden die durch unterschiedliche Standard-Präparationen ausgelösten Effekte eliminiert mit dem Resultat einer besseren Vergleichbarkeit.

Es war daher unser Ziel, unterschiedliche Methoden für die radioimmunologische Digoxin-Bestimmung dahingehend zu untersuchen, ob die beiden möglichen Erklärungen zur Verbesserung der Ergebnisse experimentell differenzierbar sind, d. h. ob eine Verbesserung der Präzision zwischen den Methoden an die Verwendung antigenarmen nativen Serums oder Plasmas als Medium zur Herstellung der Standard-Lösungen gebunden ist, oder ob die Verwendung vorbehandelter Seren oder synthetischer proteinhaltiger Pufferlösungen ebenfalls hierzu geeignet sein kann.

Als für eine derartige vergleichende Untersuchung repräsentative Methoden haben wir die drei im DigoxinRingversuch (24) am häufigsten verwendeten Methoden bzw. Testkombinationen ausgewählt. 


\section{Material und Methoden}

Verwendete Testkombinationen

A: Digoxin $\left({ }^{125} I\right)$ Radioimmunoassay Kit von Amersham Buchler

B: Radioimmunoassay Digoxin- ${ }^{125}$ I-Testgläschen an tikörperbeschichtet von Boehringer Mannheim

C: Digoxin Radioimmunoassay Kit $\left({ }^{125} \mathrm{I}\right)$ von Schwarz Mann/ Becton Dickinson

\section{Verwendete Reagenzien}

E. Merck: 5,5-Diethylbarbitursäure Natriumsalz, zur Analyse; Natriumacetat krist., zur Analyse; Natriumchlorid krist., zur Analyse; Natriumazid; Ethanol abṣolut.

Behringwerke AG: Humanserum zur Richtigkeitskontrolle Fluinorm N; Immunglobulin vom Rind, reinst; Humanalbumin, trocken, reinst.

Boehringer Mannheim: Digoxin, reinst; Digitoxin, reinst.

Tabelle 1 zeigt in einer Gegenüberștellung die wesentlichen Unterschiede in Reagenzien und Testbedingungen der drei Testkombinationen.

\section{Probenvorbereitung}

Durch Lösen von Digoxin in einer ethanolischen Lösung (12 mol/1 Ethanol im Wasser) wurde eine Lösung mit der Digoxin-Konzentration $100 \mathrm{mg} / \mathrm{l}$ hergestellt und mit Poolserum auf $1 \mathrm{mg} / \mathrm{l}$ verdünnt. Mit den Medien a, $\mathrm{b}$ und $\mathrm{c}$ wurden hieraus Verdünnungen mit den Konzentrationen 0,$25 ; 0,5 ; 1 ; 1,5 ; 2$;

$2 ; 2 ; 3 ; 4 ; 6 \mu \mathrm{g} / 1$ hergestellt, die Medien selbst wurden auch als Proben verwendet $(0 \mu \mathrm{g} / 1)$.

Medium a: Poolserum männlicher Blutspender (KontrazeptivaAusschluß)

Medium b: Humanserum Fluinorm N

Medium c: Pufferlösung mit 5,5-Diethylbarbitursäure Natriumsalz $7,13 \mathrm{mmol} / 1$; Natriumacetat $11,8 \mathrm{mmol} / 1$; Natriumchlorid $138 \mathrm{mmol} / \mathrm{l}$; Natriumazid $15,4 \mathrm{mmol} / \mathrm{l}$; Humanalbumin $50 \mathrm{~g} / \mathrm{l}$; Immunglobulin vom Rind $20 \mathrm{~g} / \mathrm{l} ; \mathrm{pH} 7,4$.

Weiterhin wurden als Proben verwendet:

Probe d: Poolserum mit zugesetztem Hämoglobin $(1,2 \mathrm{~g} / \mathrm{l})$ und Digoxin $(2 \mu \mathrm{g} / \mathrm{l})$

Probe e: Poolserum mit zugesetztem Digitoxin $(20 \mu \mathrm{g} / \mathrm{l})$

Probe f: Serum eines Patienten während Aldactone-Therapie

Proben g: Sechs Seren, die durch Verdünnung von Poolserum mit isotoner Natriumchloridlösung in den Verhältnissen $1: 1,5 ; 1: 2 ; 1: 4 ; 1: 8 ; 1: 16 ; 1: 32$ hergestellt wurden; durch Zusatz von Digoxin wurde die Konzentration dieser Proben auf $2 \mu \mathrm{g} / \mathrm{l}$ eingestellt.

Proben h: Zehn Serumproben aus einem pharmakokinetischen Versuch mit Digoxin; żwềi Seren wurden vor, acht Seren zu verschiedenen Zeiten nach Digoxin-Gabe gewonnen.
Die Proben wurden in 1 ml-Portionen vereinzelt, tiefgefroren und erst zum Zeitpunkt der jeweiligen Bestimmung aufgetaut.

\section{Durch führung}

Alle Tests wurden ohne Modifikationen der Arbeitsanleitungen von nur einem Techniker durchgeführt. Die Messung der Proben und Standards erfolgte in Triplikaten, unspezifische Bindungen wurden für jeweils eine Probe der Medien $a, b$ und $c$ sowie für jede weitere der Proben ebenfalls in Triplikaten ermittelt. Bei der Messung der Radioaktivität wurde jede Probe bis zu einer Impulssumme von 10.000 ausgezählt.

\section{Berechnung der Ergebnisse}

Aus den gemessenen Impulsraten wurden für jede der Testkombinationen entsprechen deren Arbeitsanleitung die Konzentrationswerte für die Proben jeweilig unter Bezug auf folgende Standardkurven berechnet:

\section{Standardkurve der Testkombination}

II. Proben in Medium a (Wiederfindekurve in Poolserum)

III. Proben in Medium b (Wiederfindekurve in Fluinorm N)

IV. Proben in Medium c (Wiederfindekurve in Albumin/Globulin-Puffer)

Sämtliche in dieser Weise durchgeführten Berechnungen wurden mit den für die individuellen unspezifischen Bindungen korrigierten Werten wiederholt und im Ergebnisteil 4. zur Demonstration des Einflusses der unspezifischen Bindungen verwendet.

Mit den Konzentrationswerten wurden folgende Berechnungen vorgenommen:

1. . Ermittlung von intra-assay Präzisionen für jede der Testkombinationen A, B und C

1.1. Regressionen zwischen zugesetzten und wiedergefundenen Digoxin-Konzentrationen für die Medien a, b und $c$

1.2. Mittlere Wiederfindung über den Konzentrations-Bereich $0,5-4 \mu \mathrm{g} / 1$ für die Medien a, b und c

1.3. Vergleich der Präzisionen für Proben vom Typ h (VK aus $\bar{x} \pm s$ über die Konzentrationswerte, die aus dem jeweiligen Bezug auf II, III und IV resultieren) mit der Präzision über jeweilig konzentrations-analoge Proben in den Medien a, $b$ und $c$ (VK aus $\bar{x} \pm s$ über die Konzentrationswerte, die aus dem Bezug auf $I$ resultieren)

1.4. Intra-assay Präzision für drei identische Proben mit einer Digoxin-Konzen tration von $2 \mu \mathrm{g} / \mathrm{l}$

2. Ermittlung der inter-assay Präzision zwischen den Testkombinationen $\mathrm{A}, \mathrm{B}$ und $\mathrm{C}$

2.1. Einfluß der Serumprotein-Konzentration, der Hämolyse, des Digitoxins und von Spironolacton-Metaboliten

2.2. Vergleich der Medien der Standardkurven I bis IV (VK aus $\bar{X} \pm s$ über die Konzentrationswerte aus dem jeweiligen Bezug auf I, II, III oder IV für Proben der Typen $d$ und $h$ )

3. Vergleich der Bindungen des markierten Antigens an den Antikörper in unterschiedlichen Medien

4. Einfluß $\dot{B}$ der individuellen unspezifischen Bindungen

Tab. 1. Übersicht zu Reagenzien und Testbedingungen der Testkombinationen A, B und C.

\begin{tabular}{|c|c|c|c|c|c|c|}
\hline $\begin{array}{l}\text { Test- } \\
\text { kombi- } \\
\text { nation }\end{array}$ & Medium der Standards & Trenntechnik & $\begin{array}{l}\text { Probe- } \\
\text { volumen } \\
{[\mu l]}\end{array}$ & $\begin{array}{l}\text { Ansatz- } \\
\text { volumen } \\
{[\mu l]}\end{array}$ & $\begin{array}{l}\text { Inkubations- } \\
\text { zeit } \\
\text { [min] }\end{array}$ & $\begin{array}{l}\text { Inkubations- } \\
\text { temperatur }\end{array}$ \\
\hline $\begin{array}{l}\text { A } \\
\text { B } \\
\text { C }\end{array}$ & $\begin{array}{l}\text { lyophil. Serum } \\
\text { lyophil. Serum } \\
\text { stabilisiertes Serum }\end{array}$ & $\begin{array}{l}\text { Aktivkohle } \\
\text { coated tube } \\
\text { Dextran/Aktivkohle }\end{array}$ & $\begin{array}{r}100 \\
100 \\
50\end{array}$ & $\begin{array}{r}300 \\
600 \\
1150\end{array}$ & $\begin{array}{l}30-120 \\
60 \\
30\end{array}$ & $\begin{array}{l}\text { Raumtemperatur } \\
\text { Raumtemperatur } \\
\text { Raumtemperatur }\end{array}$ \\
\hline
\end{tabular}




\section{Ergebnisse}

\section{Intra-assay Präzisionen}

für die Testkombinationen A, B und C

Durch Korrelation der den Medien a, b und c zugesetzten und wiedergefundenen Digoxin-Konzentrationen für den Bereich 0,5- $4 \mu \mathrm{g} / 1$ können bereits unterschiedliche Einflüsse der Medien auf die Ergebnisse beobachtet werden (Abb. 1.). Tabelle 2 gibt eine Zusammenstellung der entsprechenden Regressions-Gleichungen und Korrelations-Koeffizienten. Die auffäligsten Unter= schiede sind die Divergenz der Regressions-Geraden bei der Testkombination $A$, der für jedes der drei Medien a, b und c positive Ordinatenabschnitt bei der Testkombination B und der negative Ordinatenabschnitt bei der Testkombination $\mathrm{C}$.

Aus der Gegenüberstellung der mittleren Wiederf̣inderaten über den Konzentrations-Bereich von 0,5-4 $\mu \mathrm{g} / 1$ geht hervor, daß deutliche Unterschiede sowohl zwischen den Medien $\mathrm{a}, \mathrm{b}$ und $\mathrm{c}$ für jede der Testkombinationen A, B und C bestehen als auch zwischen den Testkombinationen für jedes der Medien (Tab. 3.). Für das Medium a, das in seiner Zusammensetzung der üblicherweise zu messenden individuellen Serumprobe am ähn- lichsten ist, zeigen die Testkombinationen $A$ und $C$ eine nahezu theoretische Wiederfindung des zugesetzten Digoxins mit relativ geringen Streuungen im klinisch relevanten Konzentrations-Bereich von $0,5-4 \mu \mathrm{g} / 1$, die Testkombination B zeigt eine um annähernd $15 \%$ erhöhte Wiederfindung mit relativ größerer Streuung.

Zur weiterèn Charakterisierung der vom Medium der Probe aḅhängigen, mehr oder minder ausgeprägten Störänfälligkeit der Testsysteme wurden die Streuungen der Messungen von Proben mit identischen AntigenKonzentrationen, aber mit unterschiedlichen Medien ( $a, b$ und $c$ ) für die Testkombinationen A, B und C miteinander verglichen (Tab. 4). Die Gegenüberstellung der Variationskoeffizienten für Proben mit Digoxin-Konzentrationen zwischen 0,25 und $4 \mu \mathrm{g} / 1$ läßt die Anfälligkeit der jeweiligen Testkombination gegen Medien-Unterschiède erkennen. In den niedrigen und hohen Konzentrations-Bereichen zeigt die Testkombination A relativ höhere Variationskoeffizienten, die auf die Abweichungen zurückgeführt werden können, die dạ Medium $b$ in diesem Testsystem verursacht. Dieses bestätigt die beobachtete Abweichung des Mediums b im Regressionsverhalten: Die Variationskoeffizienten für die Ergebnisse der Testkombinationen $\mathrm{B}$ und $\mathrm{C}$ sind

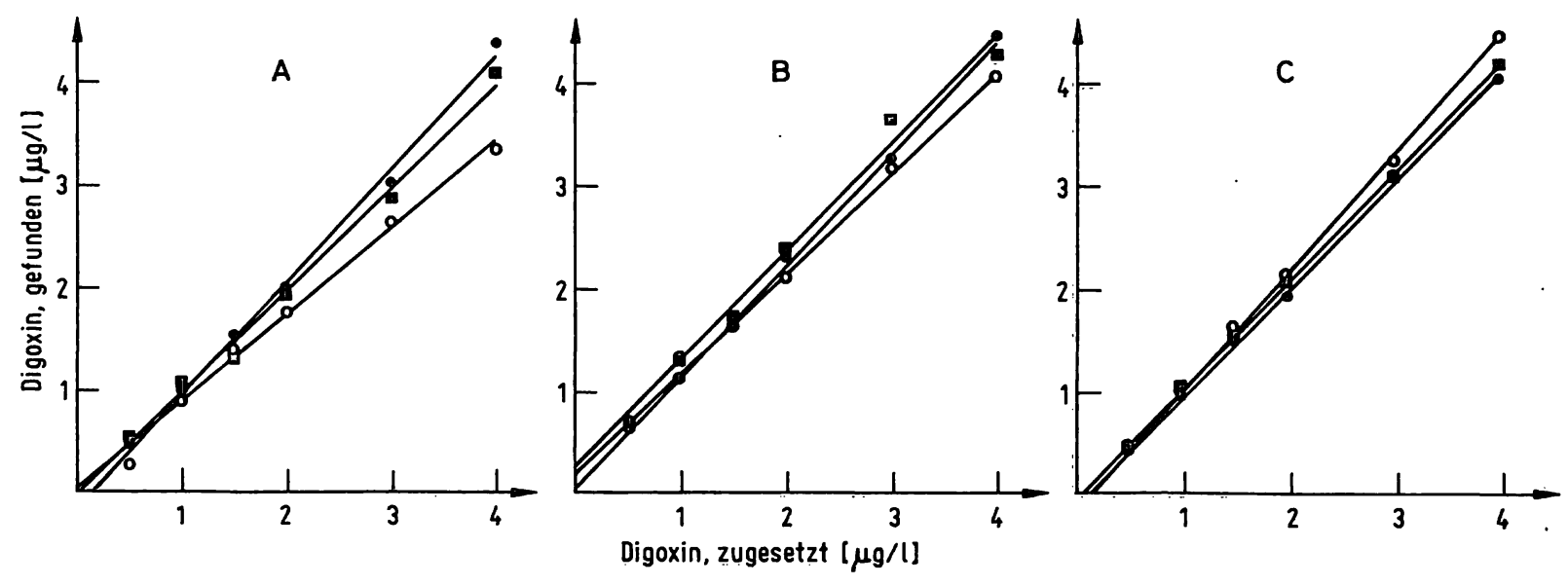

Abb. 1. Regressionen zwischen zugesetzten (Ordinaten) und gefundenen (Abszissen) Digoxin-Konzentrationen in $\mu \mathrm{g} / \mathbf{l}(\overline{\mathrm{x}} ; \mathrm{N}=3)$ in den Medien a (•), b ( 0 ) und c (a) für die Testkombinationen A, B und C.

Tab. 2. Regressionsdaten für die Korrelation $y=m \cdot x+n(y=$ Digoxin gefunden in $\mu \hat{g} / 1 ; x=$ Digoxin zugesetžt in $\mu \mathrm{g} / 1)$ für die Medien $\mathrm{a}, \mathrm{b}$ und $\mathrm{c}$ und die Testkombinationen $\mathrm{A}, \mathrm{B}$ und $\mathrm{C}$, berechnet aus je acht in Triplikaten ermittelten Konzentrationen zwischen 0,5 und $4 \mu \mathrm{g} / \mathrm{l}$.

\begin{tabular}{|c|c|c|c|}
\hline Medium & Testkombination & & \\
\hline , & $\mathbf{A}$ & B & C \\
\hline $\mathbf{a}$ & $\begin{array}{l}y=1,099 x-0,148 \\
r=0,9938\end{array}$ & $\begin{aligned} y & =1,082 x+0,087 \\
r & =0,9937\end{aligned}$ & $\begin{array}{l}y=1,031 x-0,081 \\
r=0,9983\end{array}$ \\
\hline $\mathrm{b}$ & $\begin{array}{l}y=0,852 x+0,025 \\
x=0,9907\end{array}$ & $\begin{array}{l}y=0,971 x+0,211 \\
x=0,9789\end{array}$ & $\begin{array}{l}y=1,139 x-0,132 \\
r=0,9993\end{array}$ \\
\hline c & $\begin{aligned} y & =0,989 x+0,005 \\
r & =0,9945\end{aligned}$ & $\begin{aligned} y & =1,073 x+0,200 \\
r & =0,9869\end{aligned}$ & $\begin{array}{l}y=1,052 x-0,036 \\
x=0,9992\end{array}$ \\
\hline
\end{tabular}


Tab. 3. Mittlere Wiederfindungen in Prozent $(\bar{x} \pm s ; N=24)$ für den Digoxin-Konzentrations-Bereich von 0,5 bis $4 \mu \mathrm{g} / \mathrm{l}$ für die Medien $\mathrm{a}, \mathrm{b}$ und $\mathrm{c}$ und die Testkombinationen $A, B$ und $C$.

\begin{tabular}{|c|c|c|c|c|c|c|}
\hline \multirow{2}{*}{$\begin{array}{l}\text { Me- } \\
\text { dium }\end{array}$} & \multicolumn{2}{|c|}{$\begin{array}{l}\text { Testkombination } \\
\text { A }\end{array}$} & \multicolumn{2}{|l|}{ B } & \multicolumn{2}{|l|}{ C } \\
\hline & $\overline{\mathbf{x}}$ & $s$ & $\overline{\mathbf{x}}$ & $\mathbf{s}$ & $\overline{\mathbf{x}}$ & $\mathbf{s}$ \\
\hline $\begin{array}{l}a \\
b \\
c\end{array}$ & $\begin{array}{r}100,8 \\
84,4 \\
100,2\end{array}$ & $\begin{array}{r}6,7 \\
12,6 \\
5,8\end{array}$ & $\begin{array}{l}115,9 \\
112,0 \\
119,6\end{array}$ & $\begin{array}{r}11,9 \\
16,4 \\
9,5\end{array}$ & $\begin{array}{r}98,0 \\
104,5 \\
102,4\end{array}$ & $\begin{array}{l}4,2 \\
8,1 \\
3,6\end{array}$ \\
\hline
\end{tabular}

Tab. 4. Mittlere gefundene Digoxin-Konzentrationen in $\mu \mathrm{g} / 1(\overline{\mathrm{x}})$ und Variationskoeffizienten VK $(\%)$ aus Konzentrationswerten $(N=9)$, die für konzentrations-analoge Proben in den Medien a, b und $c$ anhand der Standardkurve der jeweiligen Testkombination $(A, B, C)$ ermittelt wurden.

\begin{tabular}{llllrlrl}
\hline $\begin{array}{l}\text { Digoxin } \\
\text { zu- } \\
\text { gesetzt }\end{array}$ & \multicolumn{2}{l}{$\begin{array}{l}\text { Testkombination } \\
\text { A }\end{array}$} & & B & & C & \\
$\mu \mathrm{g} / 1$ & $\overline{\mathbf{x}}$ & $\mathrm{VK}$ & $\overline{\mathrm{x}}$ & \multicolumn{1}{c}{ VK } & $\overline{\mathbf{x}}$ & $\mathrm{VK}$ \\
\hline 0,25 & 0,16 & 69,3 & 0,44 & 26,9 & 0,20 & 25,8 \\
0,50 & 0,42 & 28,3 & 0,67 & 9,4 & 0,46 & 6,7 \\
1,00 & 1,01 & $\mathbf{8 , 0}$ & 1,23 & 14,4 & 0,99 & 5,5 \\
1,50 & 1,50 & 6,3 & 1,66 & 6,0 & 1,55 & 5,1 \\
2,00 & 1,89 & 6,4 & 2,28 & 9,1 & 2,05 & 4,3 \\
3,00 & 2,84 & 7,3 & 3,36 & 8,2 & 3,15 & 3,3 \\
4,00 & 3,94 & 12,8 & 4,28 & 6,8 & 4,21 & 4,6 \\
\hline
\end{tabular}

Tab. 5. Variationskoeffizienten VK (\%) für Replikate $(\mathrm{N}=9)$ von Proben mit einer Digoxin-Konzentration von $2 \mu \mathrm{g} / 1$ in den Medien a, $\mathrm{b}$ und $\mathrm{c}$ für die Testkombinationen $\mathrm{A}$, $B$ und $C$.

\begin{tabular}{llrl}
\hline $\begin{array}{l}\text { Medium } \\
\text { der Proben }\end{array}$ & Testkombination & & \\
$\ldots$ & A & B & C \\
\hline a & 4,3 & 7,0 & 2,2 \\
b & 3,5 & 10,5 & 1,6 \\
c & 4,3 & 5,5 & 1,2 \\
\hline
\end{tabular}

nicht nur voneinander und von denjenigen der Testkombination A verschieden, alle in dieser Weise ermittelten Variationskoeffizienten sind auch deutlich und teilweise um ein Mehrfaches gegenüber denjenigen erhöht, die als intra-assay Präzișionen für Proben in jedem der Medien a, b oder c berechnet werden (Tab. 5.). Diese Differenzen lassen sich durch die Berechnung der Streuungen unter Verwendung der Proben vom Typ $h$ bestätigen, wobei die Proben der Typen a, b und $c$ jeweils als Standardkurven verwendet wurden, um die Konzentrationswerte für den Probentyp h zu ermitteln und die S̈treuung zu berechnen. Die auf diese Weise gewonnenen Variationskoeffizienten sind denjenigen gegenübergestellt, die aus $\bar{x} \pm s$ der Proben in den Medien $a, b$ und $c$ für vergleichbare Konzentrations-Bereiche berechnet werden können (Abb. 2.). In der Höhe beider Variationskoeffizienten ( 1 und 2 in Abb. 2.) werden
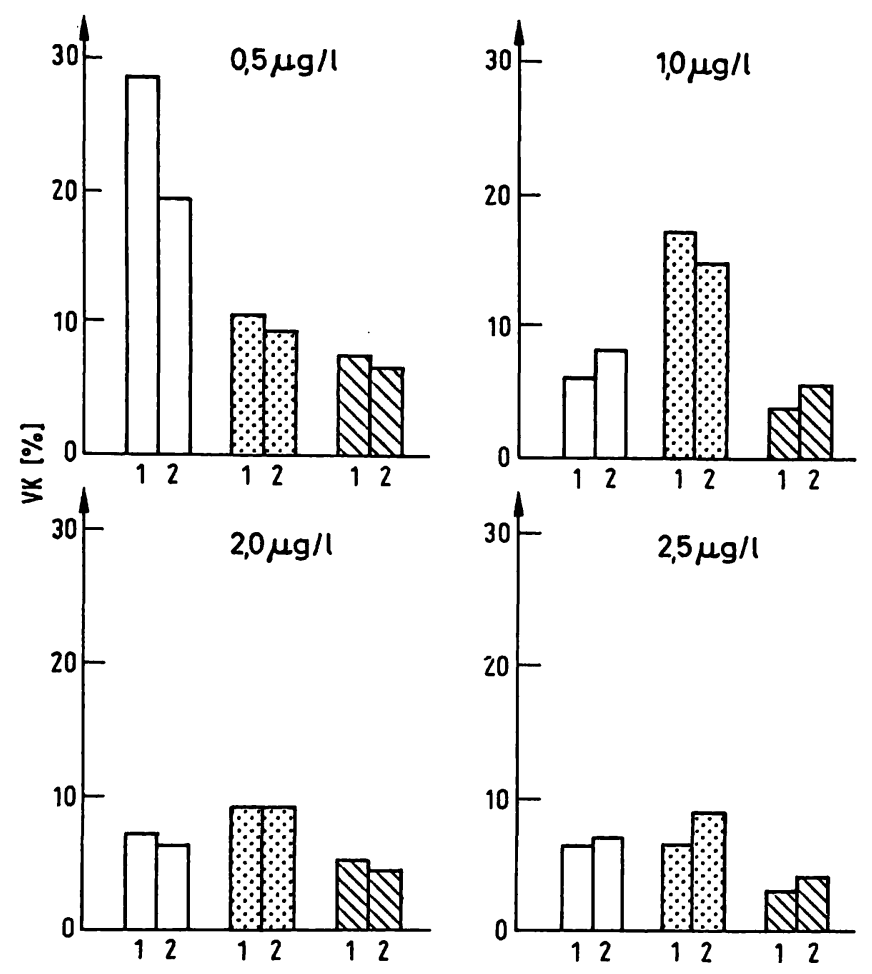

Abb. 2. Variationskoeffizienten VK (\%) aus Konzentrationswerten $(\mathrm{N}=9)$, die anhand der Standardkurven II, III und IV für Pharmakokinetik-Seren (1) ermittelt wurden und Variationskoeffizienten aus Konzentrationswerten $(N=9)$, die für konzentrations-analoge Proben in den Medien a, b und $\mathrm{c}$ anhand der Standardkurve I ermittelt wurden (2)

Testkombinationen:
A $\square$
B 因
$C \otimes$

für verschiedene Konzentrations-Bereiche bei allen drei Testkombinationen die Einflüsse der unterschiedlichen Medien gleichermaßen deutlich.

\section{Inter-assay Präzision}

$\mathrm{zw}$ ischen den Testkombinationen A, B und C

Den Einfluß der Serumprotein-Konzentration auf die gefundenen Digoxin-Konzentrationen demonstriert $\mathrm{Ab}$ bildung 3. Die Testkombinationen $\mathrm{A}$ und $\mathrm{C}$ zeigen eine Abhängigkeit des ermittelten Konzentrationswertes von der Proteinkonzentration der Probe, die allerdings erst bei derartig niedrigen Proteinkonzentrationen signifikant zu werden beginnt, wie sie auch bei pathologischen Serumproben nur selten vorkommen. Die Testkombination B zeigt diese Abhängigkeit nicht, aber eine PräzisionsErniedrigung.

Einen Uberblick zu den weiteren die Ergebnisse beeinflussenden Faktoren gibt die Tabelle 6. Hämolyse mittleren Grades bewirkt keine Störungen bei den Testkombinationen $A$ und $C$, mit $B$ wird auch nach Korrektur für die erhöhte Wiederfindung eine geringfügig höhere Digoxin-Konzentration gefunden. Die Kreuzreaktionen mit Digitoxin und Metaboliten des Spironolactons sind in Abhängigkeit von der jeweiligen Antikörper-Spezifität 


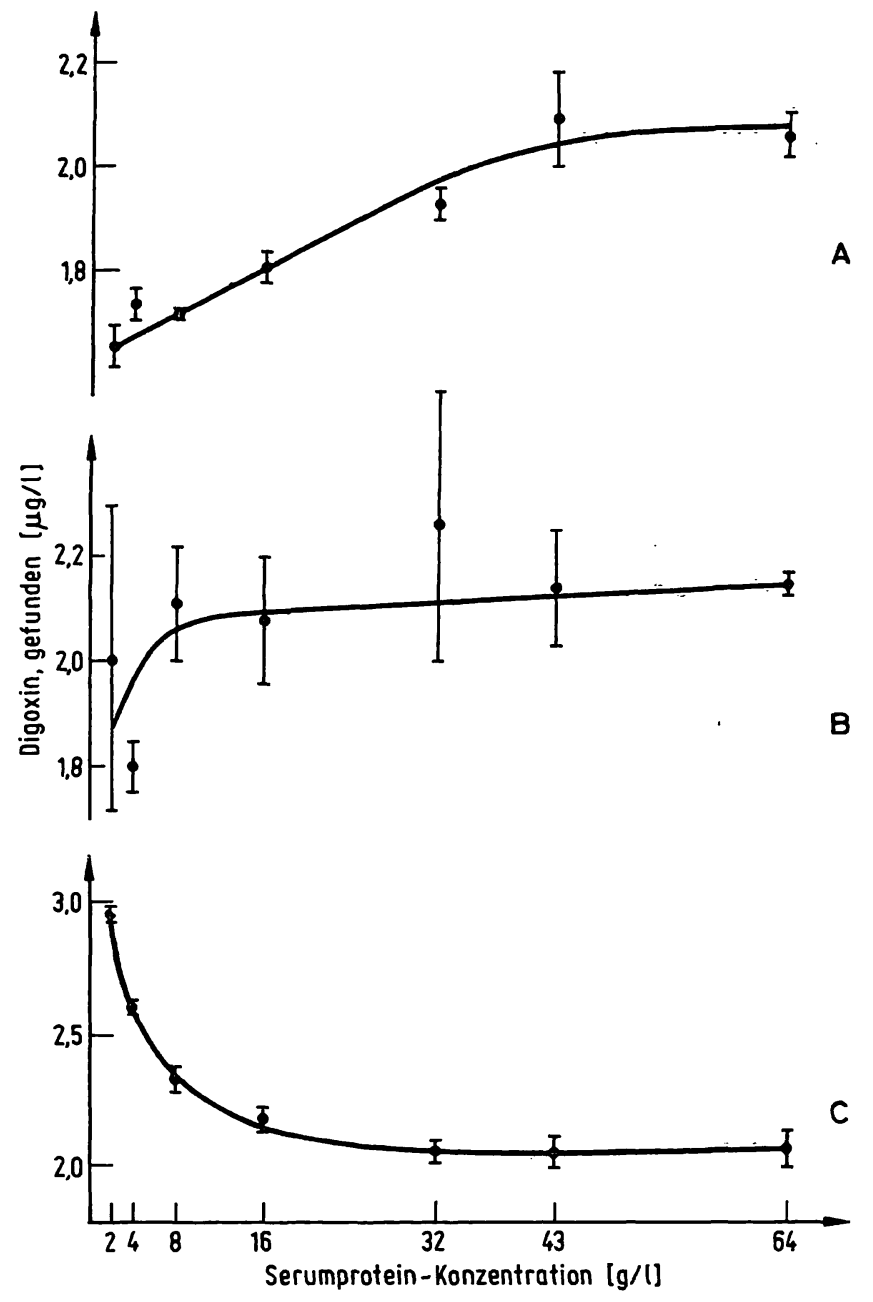

Abb. 3. Abhängigkeit der gefundenen Digoxin-Konzentrationen in $\mu \mathrm{g} / 1(\overline{\mathrm{x}} \pm \mathrm{s} ; \mathrm{N}=3)$ vom Proteingehalt in Serum-Verdünnungen mit $2 \mu \mathrm{g}$ Digoxin/1 für die Testkombinationen $A, B$ und $C$.
Tab. 6. Mittlere „Digoxin“-Konzentrationswerte $(\dot{\bar{x}})$ und Streuungen (s) in $\mu \mathrm{g} / \mathrm{l}$ von Serumproben mit Hämolyse (Hämoglobin zugesetzt: 1,2 g/l; Digoxin zugesetzt: $2 \mu \mathrm{g} / \mathrm{l}$ ), Digitoxin $(20 \mu \mathrm{g} / \mathrm{l}$ zugesetzt) und Spironolacton-Metaboliten für die Testkombinationen A, B und C.

Die Werte in Klammern sind nach subtraktiver Korrektur für die individuellen unspezifischen Bindungen berechnet worden.

\begin{tabular}{lrllllllll}
\hline & \multicolumn{3}{l}{ Testkombination } & & & \\
& $\mathrm{A}$ & & $\mathrm{B}$ & & $\mathrm{C}$ & \\
& $\overline{\mathbf{x}}$ & $\mathrm{s}$ & & $\overline{\mathbf{x}}$ & $\mathrm{s}$ & & $\overline{\mathbf{x}}$ & $\cdots$ & $\mathrm{s}$ \\
\hline Hämolyse & 2,05 & 0,08 & 2,43 & 0,08 & 2,01 & 0,04 \\
& $(1,93$ & $0,06)$ & $(2,40$ & $0,09)$ & $(2,01$ & $0,04)$ \\
Digitoxin & 1,09 & 0,03 & 2,80 & 0,07 & 1,07 & 0,03 \\
& $(1,04$ & $0,03)$ & $(2,77$ & $0,07)$ & $(1,07$ & $0,03)$ \\
Spironolacton- & 0,67 & 0,01 & 0,04 & 0,05 & 0,20 & 0,03 \\
Metabolite & $(0,64$ & $0,01)$ & $(0,05$ & $0,04)$ & $(0,21$ & $0,02)$ \\
\hline
\end{tabular}

verschieden. Während die Antikörper der Testkombinationen $\mathrm{A}$ und $\mathrm{C}$ in merklichem Ausmaße sowohl mit Digitoxin als auch mit Spironolacton-Metaboliten reagieren, zeigt der Antikörper der Tëstkombination B nur eine Kreuzreaktion mit Digitoxin, nicht mit Spironolacton-Metabolițen.

Zur Bèrechnung der inter-assay Präzision aus den Konzentrationswèrten, die durch Beżug auf unterschiedliche Standardkurven (I-IV) ermittelt wurden, dienten neun Proben der Typen d (hämolytisches Serum) und h (acht Proben aus dem pharmakokinetischen Versuch). In der Abbildung 4 sind die Streuungen aus $\bar{x} \pm s$ für jede der Proben als Variationskoeffizient angegeben und für die vier unterschiedlichen Standardkurven einander gegenübergestellt. Die interassay Präzision, die sich auf der Basis der Standardkurven der Testkombinationen (I) berechnen läßt, kann durch die Verwendung der Standardkurven in den Medien a, b oder c (entsprechend II, III oder IV) jeweils in gleicher Weise um annähernd das Doppelte verbessert werden, wobei keines der Medien gegenüber dẹm anderen als bevorzugt geeignet zu bezeichnen ist.

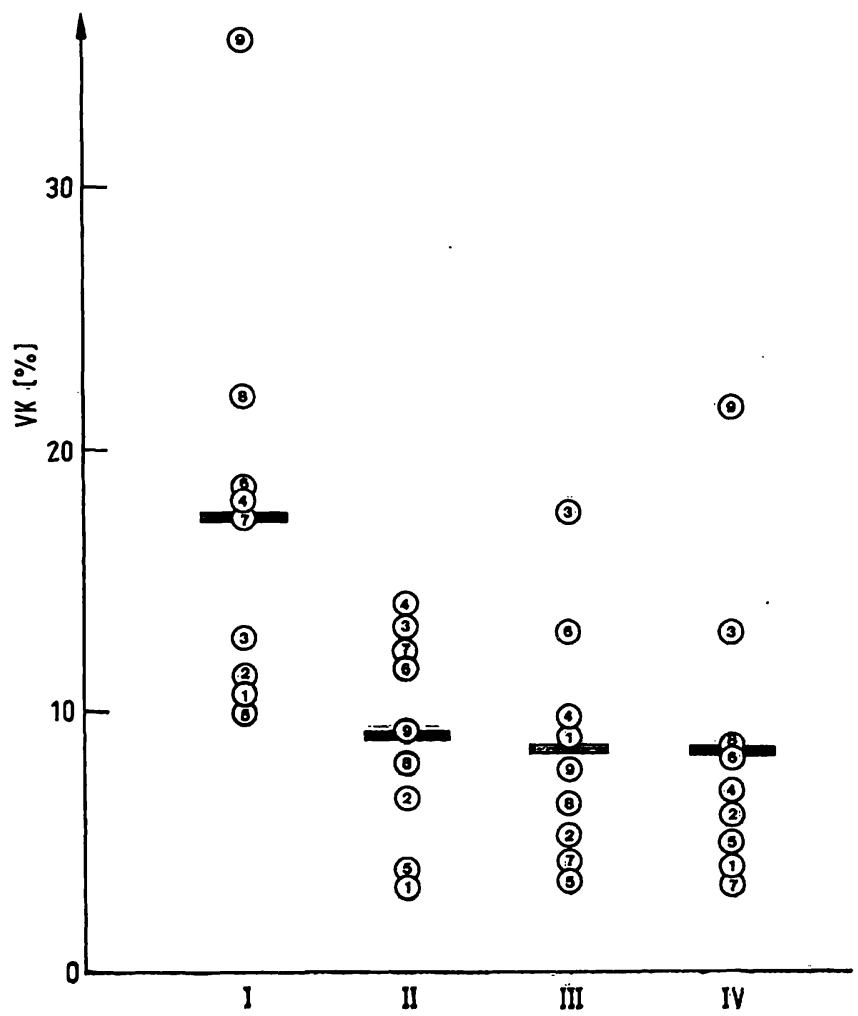

Abb. 4. Inter-assay Präzision zwischen den Testkombinationen A, B und C. Variationskoëffiżienten VK (\%) aus Konzentrationswerten $(N=9)$, die anhand der Standardkurven I bis IV fur neun Serumproben $(1)$ bis () ermittelt wurden.

- = mittlerer Variationskoeffizient.

Bezugs-Standardkurve I: Testkombination

II: Medium a

III: Medium b

IV: Medium c 
Tab. 7. Mittlere „Digoxin“-Konzentrationswerte in $\mu \mathrm{g} / \mathrm{l}(\overline{\mathbf{x}})$ für zwei digitalisfreie Serumproben (h1 und h2), ermittelt mit den Testkombinationen A, B und C durch jeweiligen Bezug auf die Standardkurven I bis IV. $\Delta=$ Differenz zwischen höchster und niedrigster ,Kọzentration"

\begin{tabular}{|c|c|c|c|c|c|c|c|c|c|}
\hline \multirow[t]{2}{*}{ Probe } & \multirow[t]{2}{*}{$\begin{array}{l}\text { Test- } \\
\text { kombination }\end{array}$} & \multicolumn{2}{|c|}{$\begin{array}{l}\text { Standardkurven } \\
\text { I }\end{array}$} & \multicolumn{2}{|l|}{ II } & \multicolumn{2}{|l|}{ III } & \multicolumn{2}{|l|}{ IV } \\
\hline & & $\overline{\mathbf{x}}$ & $\Delta$ & $\overline{\mathbf{x}}$ & $\Delta$ & $\overline{\mathbf{x}}$ & $\Delta$ & $\bar{x}$ & $\Delta$ \\
\hline $\mathrm{h} 1$ & $\begin{array}{l}\mathbf{A} \\
\mathbf{B} \\
\mathbf{C}\end{array}$ & $\begin{array}{r}-0,08 \\
0,34 \\
-0,15\end{array}$ & 0,49 & $\begin{array}{r}-0,02 \\
0,17 \\
-0,04\end{array}$ & 0,21 & $\begin{array}{r}0,01 \\
0,24 \\
-0,05\end{array}$ & 0,29 & $\begin{array}{r}-0,21 \\
0,06 \\
-0,14\end{array}$ & 0,27 \\
\hline $\mathrm{h} 2$ & $\begin{array}{l}\text { A } \\
\text { B } \\
\text { C }\end{array}$ & $\begin{array}{r}-0,09 \\
0,06 \\
-0,12\end{array}$ & 0,18 & $\begin{array}{l}-0,02 \\
-0,12 \\
-0,01\end{array}$ & 0,11 & 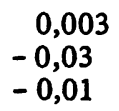 & 0,03 & $\begin{array}{l}-0,22 \\
-0,20 \\
-0,11\end{array}$ & 0,11 \\
\hline
\end{tabular}

\section{Einfluß des Mediums auf die Bindung des markierten Antigens an den Antikörper}

Für zwei Serumproben des Typs h, die Digoxin-frei sind, wurden die Konzentrationswerte über die Standardkurven I-IV für die Testkombinationen A, B und C ermittelt (Tab. 7.). Die negativen Vorzeichen resultieren daraus, daß die Bindungswerte, die für diese Seren ermittelt wurden, höher als die Bindungswerte der jeweiligen Bezugs-Standardkurve liegen. Durch Extrapolation der Standardkurven und Ablesung wurden die fiktiven Konzentrationswerte im negativen Bereich der Abszisse ermittelt. Die $\Delta$-Werte der Tabelle 7 sind die vorzeichenlosen Differenzen zwischen dem höchsten und dem niedrigsten Konzentrationswert; sie stellen jeweils die maximalen inter-assay Differenżen für die vier verschiedenen Standardkurven dar. Für beide Proben ist eine deutliche Verminderung dieser Differenzen zu beobachten, wenn anstelle der Standardkurven I der Testkombinationen die Standardkurven II, III oder IV als Berechnungsgrundlage verwendet werden. Aus den in Abhängigkeit von der Kombination Testkit/Standardkurven-Medium resultierenden unterschiedlichen ,negativen“ bis positiven Konzentrationswerten für Seren ohne Digoxin-Gehalt geht hervor, daß die Ergebnisse durch Serumfaktoren beeinflußt werden, die einer weiteren Charakterisierung bedürfen.

\section{Bedeutung der individuellen unspezifischen Bindungen}

Zur Demonstration des Einflusses der unispezifischen Bindungen wurden mittlere Konzentrationswerte mit Standardabweichungen, die sich ohne Berücksichtigung der unspezifischen Bindungen berechnen lassen, solchen gegenübergestellt, die nach vorhergehender subtraktiver Korrektur der individuellen unspezifischen Bindungen ermittelt wurden (Tab. 6.). Für die mit den Testkombinationen A, B und C untersuchten Probentypen konnte kein Einfluß der unspezifischen Bindungen auf die Mittelwerte mit Streuungen festgestellt werden, da die unspezifischen Bindungen für Standards und Proben identisch sind.

\section{Diskussion}

Die insbesondere von Röhle et al (32), Breuer et al (33) und Marschner et al $(25-29,31,33)$ ausgehenden Bemühungen um die Qualitätskontrolle bei radioimmunologischen Analysenverfahren in der Bundesrepublik Deutschland haben zu zwei inzwischen etablierten Konzeptionen für die Durchführung von Ringversuchen gefuihrt. Der eine Typus sieht die Messung von etwa 20 tiefgefrorenen Proben vor, die u. a. eine Wiederfindekurve von Standard-Antigen in antigenarmem oder -freiem Serum en thalten. Dieses Vorgehen wurde auch einem Digoxin-Ringversuch (24) zugrundegelegt und dabei das aus Ringversuchen für Hormone $(25,26$, 28-33) bereits früher gefundene Resultat bestätigt, daß die inter-assay Präzision bei Verwendung derartiger Wiederfindekurven erheblich verbessert werden kann.

Vergleichende Untersuchungen von Methoden können unter bestimmten Voraussetzungen besser als Ringversuche geeignet sein, Unterschiede zwischen den Methoden zu erkennen, da Ringversuche neben allen anderen Fehlern, die zu Streuungen beitragen, als zusätzliche Fehlermöglichkeit die interindividuellen Fehler enthalten. Bei einem Vergleich von Methoden, der von nur einem erfahrenen Untersucher durchgeführt wird, ist der relativ größere interindividuelle Fehler durch den relativ geringeren intraindividuellen Fehler ersetzt und erkennbare Differenzen in den Ergebnissen sind eindeutiger den methodischen Unterschieden zuzuordnen.

Bei der Messung von Proben mit identischen DigoxinKonzentrationen in unterschiedlichen Medien werden sowohl im intra- als auch im inter-assay Vergleich bei drei verschiedenen Testkombinationen Differenzen in den Ergebnissen beobachtet. Durch die jeweiligen Korrelationen von in drei unterschiedlichen Medien ermittelten Konzentrationen mit den Sollwerten kommen in den Konstanten der Regressions-Geraden Abweichungen zum Ausdruck, die durch Einflüsse der jeweiligen Medien gegenüber denjenigen der Kit-Standards resultieren. Derartige Differenzen sind auch zwischen den Antigen- 
Antikörper-Bindungen in Digoxin-freien Seren in Abhängigkeit von der Art der Bezugs-Standardkurve und der Testkombination zu beobachten. Da jedoch im Gegensatz zur radioimmunologischen Insulin-Bestimmung (38) bei den untersuchten Testkombinationen zur Digoxin-Bestimmung jeweils die unspezifischen Bindungen für Standards und Proben identisch sind, können die intra- und inter-assay Unterschiede nicht durch die Berücksichtigung der unspezifischen Bindungen verringert werden.

Die Ergebnisse zur Wiederfindung von Digoxin in Poolserum sowie zum Einfluß der Hämolyse entsprechen denjenigen, die auch durch den Digoxin-Ringversuch (24) ermittelt wurden. Die für alle drei Testkombinationen beobachteten Effekte, die durch unphysiologisch niedrige Serumprotein-Konzentrationen hervorgerufen werden, verdeutlichen die Notwendigkeit einer physiologischen Protein-Konzentration für_Digoxin-Kontrollseren. Dieses Kriterium wird von den kommerziell vertriebenen Präparationen nicht immer erfüllt.

Die von der Spezifität der Antikörper abhängigen Ergebnisse sind für zwei der drei Testkombinationen nicht mit denjenigen des Digoxin-Ringversuches (24) vergleichbar, da zwischenzeitlich die Antikörper-Chargen gewechselt worden sind. Während die Kreuzreaktionen mit Digitoxin gegenüber den Ringversuchs-Ergebnissen für die Testkombination B von 0,98 auf $2,80 \mu \mathrm{g} / 1$, Digoxin" zugenommen und für die Testkombination $\mathrm{C}$ von 1,72 auf $1,07 \mu \mathrm{g} / 1$,Digoxin“ abgenommen hảben, sind demgegenüber gleichzeitig die Reaktivitäten mit Spironolacton-Metaboliten bei der Testkombination B von 0,28 auf $0,04 \mu \mathrm{g} / 1$,Digoxin" vermindert, bei der Testkombination $\mathrm{C}$ von 0,05 auf $0,20 \mu \mathrm{g} / 1$, Digoxin“ erhöht. Dieses bestätigt den bereits im Ringversuch erkennbaren Zusammenhang, daß für ein definiertes Antiserum eine hohe Kreuzreaktivität mit Digitoxin mit einer niedrigen Kreuzreaktivität mit SpironolactonMetaboliten gekoppelt zu sein scheint und umgekehrt.
Als ein im Rahmen der Qualitätskontrolle wesentliches Problem haben wir versucht, zu ermitteln, ob bei der Digoxin-Bestimmung zur Steigerung der interassay Präzision die Verwendung nativen Serums als Milieu für die Wiederfindekurve der Verwendung artefizieller oder synthetischer Medien überlegen ist. Dieses ist, wie die Ergebnisse zeigen, nicht der Fall. Man kann daher annehmen, daß die Präzisions-Verbesserung bei der Digoxin-Bestimmung nicht auf der Verwendung antigenfreien Serums beruht, sondern auf dem Charakter der Einheitlichkeit der Bezugs-Standardkurve. Da DigoxinBestimmungen in der Bundesrepublik Deutschland nahezu ausschließlich mit auch im. Ausland konfektionierten Testkombinationen durchgeführt werden, ist das Problem der Verbesserung der inter-assay Präzision unmittelbar mit den Interessen der Hersteller von Testkombinationen an Problemen der externen Qualitätskontrolle verbunden. Im gegenwärtigen Stadium der Qualitätskontrolle radioimmunologischer Bestimmungen gibt es offenkundig nur begrenzte Möglichkeiten, eine Verbesserung der Vergleichbarkeit der Ergebnisse durch eine Normierung von Standardpräparationen zu erreichen. Ein Weg führt über die einheitliche Verwendung identischer Standardpräparationen; dieses bedarf jedoch eines erheblichen organisatorischen Aufwandes oder entsprechender legislativer Maßnahmen. Eine zweite Möglichkeit ist die für die Hormon-Bestimmungen von Marschner et al (25-29, 33-35) realisierte Empfehlung zur Verwendung von antigenarmen Seren zur Herstellung der Bezugs-Standardkurve. Wir konnten zwar demonstrieren, daß bei der Digoxin-Bestimmung die Verwendung weiterer Medien in gleicher Weise den gewünschten Effekt zeigt, wir möchten aber dennoch ebenfalls die Verwendung frischen Mischserums männlicher Blutspender zur Herstellung uniformer BezugsStandardkurven empfehlen, da wir meinen, daß ein derartiges Mischserum in seiner Zusammensetzung langfristig konstanter sein wird als Reagenzien, die zwangsläufig einem mehr oder weniger häufigen Chargenwechsel unterliegen.

\section{Literatur}

1. Smith, T. W., Butler, Jr., V. P. \& Haber, E. (1969), N. Engl. J. Med. 281, 1212-1216.

2. Belpaire, F. M., Bogaert, M. G. \& De Broe, M. E. (1975), Clin. Chim. Acta 62, 255-261.

3. Battaglia, D. J. \& Cianci, M. L. (1976), J. Nucl. Med. 17, $847-849$.

4. Müller, H., Graul, E. H. \& Müller, L. (1975), Z. Kardiol. $64,1123-1139$.

5. Reisert, P. M. \& Kistner, M. (1977), Fortschr. Med. '9'5, $167-171$

6. Kramer, P., Saul, J., Köthe, E. \& Scheler, F. (1975), Klin. Wochenschr. 53, 215-219.

7. Kubasik, N. P., Norkus, N. S. \& Sine, H. E. (1974), Clin. Biochem. 7, 307-312.

8. Voshall, D. L., Hunter, L. \& Grady, H. J. (1975), Clin. Chem. $21,402-406$.

9. Kuno-Sakai, H., Sakai, H. \& Ritzmann, S. E. (1975), Clin. Chem. 21, 156-157.

10. Ravel, R. (1975), Clin. Chem. 21, 1801-1803.

11. Gutcho, S., McCarter, H. \& Rapun, R. (1973), Clin. Chem 19, 1058-1059.

12. Shapiro, B., Kollmann, G. J. \& Heine, W. I. (1975), Semin. Nucl. Med. 5, 205-218.

13. Burnett, G. H., Conklin, R. L., Wasson, G. W. \& MacKinney, A. A. (1973), Clin. Chem. 19, 725-726.

14. Kuno-Sakai, H. \& Sakai, H. (1975), Clin. Chem. 21, 227229.

15. Kuczala, Z. J. \& Ahluwalia, G. S. (1976), Clin. Chem. 22, 193-197.

16. Barbieri, U. \& Gandolfi, C. (1977), Clin. Chim. Acta 77, 257-267.

17, Kubasik, N. P., Hall, J. L., Barold, S. S., Volosin, M. T. \& Sine, H. E. (1976), Chest 70, 217-220.

18. Taubert, K. \& Shapiro, W. (1975), Am. Heart J. 89, 79-86.

19. Kubasik, N. P., Hall, J. L. \& Sine, H. E. (1976), Clin. Chem. $22,1745-1746$. 
20. Kubasik, N. P., Brody, B. B. \& Barold, S. S. (1975), Am. J. Cardiol. 36, 975-977.

21. Meade, R. C. \& Kleist, T. J. (1972), J. Lab. Clin. Med. 80, 748-754.

22. Wood, W. G. (1977), diese Z. 15, 679-685.

23. Boink, A. B. T. J., Kruyswijk, H. H., Willebrands, A. F. \& Maas, A. H. J. (1977), diese Z. 15, 261-266.

24. Dwenger, A., Friedel, R. \& Trautschold, I. (1978), Performance of radioimmunoassays for digoxin as evaluated by a group experiment, in: Proceedings, International symposium on radioimmunoassay and related procedures in medicine, Berlin 1977, International Atomic Energy Agency, Vienna, im Druck.

25. Marschner, I., Bottermann, P., Erhardt, F., Linke, R., Löffler, G., Maier, V., Schwandt, P., Vogt, W. \& Scriba, P. C. (1974), Horm. Metab. Res. 6, 293-296.

26. Marschner, 1., Biro, G., Erhardt, F. W., Scriba, P. C. \& Weinges, K. (1975), Informationsblatt der Deutschen DiabetesGesellschaft Nr. 3, 1-9.

27. Erhardt, F., Marschner, I., Pickardt, R. C. \& Scriba, P. C. (1973), diese Z. 11, 381-387.

28. Marschner, I., Erhardt, F. W. \& Scriba, P. C. (1976), diese Z. $14,345-351$.

29. Horn, K., Marschner, I. \& Scriba, P. C. (1976), diese Z. 14, 353-360.

30. Gordon, A. \& Gross, J. (1976), Acta Endocrinol. 83, 539548.
31. Wood, W. G., Marschner, I. \& Scriba, P. C. (1978), The need for standardization of methodology and components in commercial radioimmunoassay kits, in: Proceedings, International symposium on radioimmunoassay and related procedures in medicine, Berlin 1977, International Atomic Energy Agency, Vienna, im Druck.

32. Röhle, G., Breuer, H. \& Oberhoffer, G. (1975), Dtsch. Ärzteblatt $72,883-890$.

33. Breuer, H., Jungbluth, D., Marschner, I., Röhle, G., Scriba, P. C. \& Wood, W. G. (1978): The current state of external quality control surveys in the German Federal Republic in the field of peptide hormone radioimmunoassays, in: Proceedings, International symposium on radioimmunoassay and related procedures in medicine, Berlin 1977, International A tomic Energy Agency, Vienna, im Druck.

34. Erhardt, F. W. \& Scriba, P. C. (1974), Ärztl. Lab. 20 , 191-198.

35. Erhardt, F. W., Marschner, I. \& Scriba, P. C. (1975), diese Z. 13, 249-250.

36. Cresswell, M. A., Hall, P. E. \& Hurn, B. A. L. (1978), Quality control in RIA - a report on the results of an external quality control programme in a group of 140 laboratories, in: Proceedings, International symposium on radioimmunoassay and related procedures in medicine, Berlin 1977, International A tomic Energy Agency, Vienna, im Druck.

37. Stamm, D. \& Büttner, H. (1969), diese Z. 7, 393-403.

38. Dwenger, A., Tost, P. \& Trautschold, I. (1977), diese Z. 15, 593-602.

Dr. rer. nat. A. Dwenger Institut für Klinische Biochemie der Medizinischen Hochschule Karl-Wiechert-Allee 9

D-3000 Hannover 61 
. 